International Journal of Applied Mathematics

Volume 28 No. $3 \quad 2015,253-273$

ISSN: 1311-1728 (printed version); ISSN: 1314-8060 (on-line version)

doi: http://dx.doi.org/10.12732/ijam.v28i3.6

\title{
PARAMETER HOMOTOPY CONTINUATION FOR FEEDBACK LINEARIZATION OF NON-REGULAR CONTROL-AFFINE NONLINEAR SYSTEMS
}

\author{
Alex Borisevich $^{1}$, Gernot Schullerus ${ }^{2}$ \\ ${ }^{1}$ Samsung SDI R\&D Center \\ 467 Beonyeong-ro, Seobuk-gu, Cheonan-si, 331-300, KOREA \\ 2 Reutlingen University \\ Alteburgstr. 150, Reutlingen, 72762, GERMANY
}

\begin{abstract}
In this article feedback linearization for control-affine nonlinear systems is extended to systems where linearization is not feasible in the complete state space by combining state feedback linearization and homotopy numerical continuation in subspaces of the phase space where feedback linearization fails. Starting from the conceptual simplicity of feedback linearization, this new method expands the scope of their applicability to irregular systems with poorly expressed relative degree. The method is illustrated on a simple SISOsystem and by controlling the speed and the rotor flux linkage in a three-phase induction machine.
\end{abstract}

AMS Subject Classification: 93B18, 93C10, 65H20

Key Words: non-regular control-affine systems, homotopy continuation, feedback linearization, ill-defined relative degree

\section{Introduction}

The control of affine nonlinear systems has been extensively studied and several control methods like feedback linearization [13], differential smoothness-based methods [12, 18], Lyapunov functions and its generalizations [23], including a backstepping [16] as well as sliding mode control [22] and approximation of

Received: April 2, 2015

(c) 2015 Academic Publications

${ }^{\S}$ Correspondence author 
smooth dynamic systems by hybrid switching systems and hybrid control [3] have been developed. Although the applicability and practical implementation has been repeatedly confirmed in laboratory tests and in commercial hardware, the improvement of these control techniques is still an active area of research.

The paper presents a homotopy-based variant of feedback linearization in order to deal with regularity issues that occur if the input/output coupling matrix looses rank. The problem of ill-defined relative degree is well-known in literature. For example, most underactuated systems are not fully feedback linearizable, and smooth feedback stabilization to a single equilibrium point is not possible $[10,14,15,1]$. Another case of the nonlinear system with ill-defined relative degree consideredm is DC-motor in bidirectional operation when the armature current approaches to zero [24]. Similar situations occur in other electrical machines, depending on the operating mode and the model parametrization [6].

In the case where the input-output decoupling matrix is rank-degenerate, the controller can be obtained by direct application of the dynamic extension algorithm [20]. It has been shown, that for a system which is not feedback linearizable, the addition of integrators in the inputs can result in the higher order system being feedback linearizable. However such procedure could be implemented only when decoupling matrix has constant rank. The presence of singularities is a fundamental limitation of dynamic extension algorithm that relies on the decomposition of input-output dependence at controller synthesis stage [17].

One of the main approaches to this problem is to use a switching control scheme [9], that is, a tracking control law that switches between an approximate input-output linearization control law when the state is close to the singularities and an exact input-output linearization control law when the state is far from the singularities. In one of recent publications [14] the applicability of a composite control scheme consisting of heuristic-based approximate linearization and backstepping design for a nonlinear system is investigated. Another type of solution is presented in [1] where inverse Lyapunov approach in conjunction with the energy shaping technique is applied to ball and beam system. There are approaches that allow steering through a singular point (loss of regularity) using a time scaling of the reference trajectory which would allow one to stay with the standard feedback linearization approach [15].

Interestingly, in the numerical methods for solving systems of nonlinear equations, the problem of ill-defined relative degree has a similar counterpart, the singularity of the Jacobian. And one of the most powerful method for overcoming problems related with singular points and initial solution guess is 
numerical parameter continuation [2]. This paper continues the work of the authors $[8,7]$ on the application of homotopy numerical continuation methods to control problems. The major novelty presented here is a control approach for driving output to zero which is suitable for MIMO systems with a relative degree more than one. During control action algorithm switches to feedback linearization only near the origin, where homotopy continuation stage is over and system output is close to the desired state.

The immediate motivation for using the parameter continuation method in control problems is a series of papers $[4,5]$, where the application of these methods is described in combination with physical experiments.

In order to illustrate the method, we will first briefly introduce the control problem and review feedback linearization as a possible solution to the given problem. Then, an example illustrates the limitation of feedback linearization. The new method is presented subsequently and its application is illustrated by examples.

\section{Problem Statement}

In this paper we consider control-affine nonlinear systems and solve the problem of designing a controller which drives the system output asymptotically to the equilibrium point 0 .

Problem 1. Given the control-affine nonlinear system

$$
\begin{gathered}
\dot{x}=f(x)+\sum_{i=1}^{m} g_{i}(x) u_{i}, \quad y=h(x), \\
x \in X \subseteq \mathbb{R}^{n}, \quad y \in Y \subseteq \mathbb{R}^{m}, \quad u \in U \subseteq \mathbb{R}^{m}, \\
f: \mathbb{R}^{n} \rightarrow \mathbb{R}^{n}, \quad g_{i}: \mathbb{R}^{n} \rightarrow \mathbb{R}^{n}, \quad h: \mathbb{R}^{n} \rightarrow \mathbb{R}^{m}, \\
f, g, h \in C^{k},
\end{gathered}
$$

where $f, g, h$ - are sufficiently smooth functions. Find an appropriate statefeedback control law $u(x)$ the application of which drives the system output $y$ to 0 .

One method for solving this problem is feedback linearization. It is reviewed in the sequel. 


\subsection{Feedback Linearization}

The main idea of feedback linearization [13] is to design a control law that leads to a linear dynamic behavior between inputs and outputs or states, respectively. When the focus is on the input-output-relation, then the order of this dynamics is characterized by the relative degree.

Definition 1. A MIMO nonlinear system (1) has relative degree $r_{j}$ for output $y_{j}$ at point $x_{0} \in \mathbb{R}^{n}$ if following conditions satisfied:

1. $\mathcal{L}_{g_{i}} \mathcal{L}_{f}^{k} h_{j}=0$ for $k<r_{j}-1$ and any $g_{i}$ in a neighborhood of $x_{0}$,

2. for at least one function $g_{i}$

$$
\mathcal{L}_{g_{i}} \mathcal{L}_{f}^{r_{j}-1} h_{j} \neq 0
$$

where $\mathcal{L}_{f} h=\frac{\partial h(x)}{\partial x} f(x)=\sum_{i=1}^{n} \frac{\partial h(x)}{\partial x_{i}} f_{i}(x)$ is a Lie derivative of the function $h$ along a vector field $f$.

That is, at least one input $u_{k}$ influences the output $y_{j}$ after $r_{j}$ differentiations. The total relative degree $r$ of the system (1) is given by $r=\sum_{j=1}^{m} r_{j}$. If $r=n$ and the matrix

$$
A(x)=\left(\begin{array}{ccc}
\mathcal{L}_{g_{1}} \mathcal{L}_{f}^{r_{1}-1} h_{1}(x) & \cdots & \mathcal{L}_{g_{m}} \mathcal{L}_{f}^{r_{1}-1} h_{1}(x) \\
\vdots & \cdots & \vdots \\
\mathcal{L}_{g_{1}} \mathcal{L}_{f}^{r_{m}-1} h_{m}(x) & \cdots & \mathcal{L}_{g_{m}} \mathcal{L}_{f}^{r_{m}-1} h_{m}(x)
\end{array}\right)
$$

has full rank, then the original dynamical system (1) is equivalent near $x_{0}$ with respect to the input-/output behavior to the system:

$$
y_{j}^{\left(r_{j}\right)}=\mathcal{L}_{f}^{r_{j}} h_{j}+\sum_{i=1}^{m} \mathcal{L}_{g_{i}} \mathcal{L}_{f}^{r_{j}-1} h_{j} u_{i}=c_{j}^{T}(B(x)+A(x) u),
$$

where $c_{j}^{T}$ is a row vector of zeros except for the $j$-th column which is equal to 1. The nonlinear feedback

$$
u(x)=A(x)^{-1}[v-B(x)]
$$

converts in the neighborhood of $x_{0}$ the original dynamical system (1) to a linear system

$$
y^{\left(r_{j}\right)}=v_{j},
$$


which can be controlled by conventional linear control system methods. So feedback linearization methods for nonlinear systems (1) use two feedback loops, one of which implements a linearizing transformation (2) to (3), while the second one controls the system (3) using an appropriately designed linear controller.

A significant drawback which limits the applicability of the feedback linearization in practice is the requirement of a constant relative degree $r$ and full-rank of the matrix $A(x)$ in the whole phase space. This is illustrated by a simple example in the following section.

\subsection{A Simple Example}

Consider the following system

$$
\dot{x}=u, \quad y=h(x)=x\left(x^{2}-1\right)+1, \quad x(0)=1
$$

and the task of designing a control law $u=u(x)$ such that $y$ asymptotically approaches 0 .

An analysis of the system illustrates that

$$
A(x)=\mathcal{L}_{g} h=\frac{\partial h(x)}{\partial x} g(x)=3 x^{2}-1
$$

which is invertible in $x \in \mathbb{R} \backslash\left\{-\frac{1}{\sqrt{3}}, \frac{1}{\sqrt{3}}\right\}$. This means however, that feedback linearization as described in the previous subsection is not feasible in the complete state space. A similar situation is known from numerical methods for finding roots and optimization of functions with singularities. These so called homotopy continuation methods modify the search direction in the neighborhood of singularities by augmenting the system with a system with a known solution. The main idea of the approach presented in the following section is to adapt this idea to the solution of the control problem 1.

It should be noted that in this work solution proposed is a variant of dynamic controller. But compared with dynamic feedback linearization, our approach applicable to different class of systems because conditions of solution existence (14) is formulated in terms of augmented system. For the SISO plants it is known, that if the system is not linearizable by static feedback, then it also neither differentially flat, neither linearizable by dynamic state feedback [18]. Thus, for the system (4) under consideration such methods are not applicable, but homotopy-based controller can handle output zeroing problem as it shown in Section 4.1. Therefore the method proposed is applicable to wider class of SISO systems than known approach of dynamic feedback linearization. The question how related are classes of MIMO systems, to which our method is 
applicable and flatness-based or dynamic state feedback methods are working is still open.

\section{Parameter Continuation for Control-Affine Nonlinear System}

Let us associate with the plant (1) a linear dynamic system

$$
\begin{aligned}
& \dot{z}=A z+B u, \quad \eta=C z, \quad \frac{d^{\left(r_{i}\right)}}{d t^{\left(r_{i}\right)}} \eta=u_{i}, \\
& z \in X \subseteq \mathbb{R}^{n}, \quad \eta \in Y \subseteq \mathbb{R}^{m}, \quad u \in U \mathbb{R}^{m},
\end{aligned}
$$

where each output $\eta_{j}$ has the same relative degree $r_{j}$ as the original system output $y_{j}$ from (1).

In order to solve the control problem 1 we consider a new output variable $H$ given by the combination of the output variables of (1) and (6):

$$
H=(1-\lambda) \eta+\lambda y,
$$

where $\lambda \in[0,1]$ is a continuous time-dependant parameter, the value of which determines the relative contribution of the outputs $\eta$ and $y$ to $H$. The vector equation

$$
H=0
$$

defines a trajectory $(x(t), \lambda(t))$ which begins at $\left(x_{0}, 0\right)$ and ends at $\left(x^{*}, 1\right)$ where $y\left(x^{*}\right)=0$. The objective is now the design of a controller which provides a linear dynamics for $H$. Note, that in the ideal case when system model (1) is known exactly and there are no disturbances, the condition (8) is satisfied automatically due to the nature of the proposed linearization transformation.

The purpose of proposed controller is to hold $H=0$ for (7) anywhere, by adjusting of $\lambda$ and state variable $x$ simultaneously. As result, the $\lambda$ cannot reach value 1 without satisfying of condition $h\left(x^{*}\right)=0$.

Similar to conventional feedback linearization each component $H_{i}$ in (7) is differentiated $r_{i}$ times with respect to $t$ until one obtains an explicit function 
of some input $u_{j}$. We obtain after differentiation

$$
\begin{aligned}
H_{i}^{\left(r_{i}\right)}= & -\sum_{k=1}^{r_{i}-1} C_{r_{i}}^{k} \eta_{i}^{\left(r_{i}-k\right)} \lambda^{(k)}+(1-\lambda) u_{i} \\
& +\left(y_{i}-\eta_{i}\right) \lambda^{\left(r_{i}\right)}+\sum_{k=1}^{r_{i}-1} C_{r_{i}}^{k} y_{i}^{\left(r_{i}-k\right)} \lambda^{(k)} \\
& +\lambda\left(\mathcal{L}_{f}^{r_{i}} h_{i}+\sum_{k=1}^{m} \mathcal{L}_{g_{k}} \mathcal{L}_{f}^{r_{i}-1} h_{i} u_{k}\right)
\end{aligned}
$$

where $C_{n}^{k}$ are binomial coefficient. Now, the following notation is introduced:

$$
\begin{aligned}
\Lambda_{i} & =\left(\lambda, \dot{\lambda}, \ddot{\lambda}, \ldots, \lambda^{\left(r_{i}-1\right)}\right) \\
\mathcal{A}_{i, 1}(x, \lambda) & =\left(\lambda \mathcal{L}_{g_{k}} \mathcal{L}_{f}^{r_{i}-1} h_{i}\right)^{T}+(1-\lambda) c_{k}^{T} \\
\mathcal{A}_{i, 2}(x, z) & =y_{i}-\eta_{i} \\
\mathcal{B}_{i}\left(x, z, \Lambda_{i}\right) & =-\sum_{k=1}^{r_{i}-1} C_{r_{i}}^{k} \eta_{i}^{\left(r_{i}-k\right)} \lambda^{(k)}+\sum_{k=1}^{r_{i}-1} C_{r_{i}}^{k} y_{i}^{\left(r_{i}-k\right)} \lambda^{(k)}+\lambda \mathcal{L}_{f}^{r_{i}} h_{i},
\end{aligned}
$$

where $c_{k}^{T}$ is a row vector of dimension $m$, where all components except for the $k^{-}$ th component are equal to 0 . With the variables introduced in (10) equation (9) can be written more compactly:

$$
H_{i}^{\left(r_{i}\right)}=\mathcal{A}_{i, 1}(x, \lambda) u+\mathcal{A}_{i, 2}(x, z) \lambda^{\left(r_{i}\right)}+\mathcal{B}_{i}(x, z, \Lambda) .
$$

Considering all of the components $H_{i}$ after differentiation according to the relative degrees $r_{i}$ the result is a differential equation in matrix form

$$
\begin{aligned}
H^{(r)} & =\mathcal{A}_{1}(x, \lambda) u+\mathcal{A}_{2}(x, z) \lambda^{\left(r_{\max }\right)}+\mathcal{B}(x, z, \Lambda) \\
& =\mathcal{A}(x, z, \lambda)\left(\begin{array}{c}
u \\
\lambda^{\left(r_{\max }\right)}
\end{array}\right)+\mathcal{B}(x, z, \Lambda),
\end{aligned}
$$

where

$$
\begin{gathered}
r_{\max }=\max \left\{r_{i}\right\}, \quad \Lambda=\left(\lambda, \dot{\lambda}, \ddot{\lambda}, \ldots, \lambda^{\left(r_{\max }-1\right)}\right), \\
H^{(r)}=\left(H_{1}^{\left(r_{1}\right)}, H_{2}^{\left(r_{2}\right)}, \ldots, H_{m}^{\left(r_{m}\right)}\right)^{T} .
\end{gathered}
$$

Now we are ready to formulate the main result of the paper given by the following theorem: 


\section{Theorem 1. Suppose}

$$
\operatorname{rank} \mathcal{A}(x, z, \lambda)=m,
$$

then for state feedback equation

$$
\left(\begin{array}{c}
u \\
\lambda^{\left(r_{\max }\right)}
\end{array}\right)=\alpha \tau+\mathcal{A}^{+}(v-\mathcal{B}),
$$

where $v$ is the new bounded input and the vector $\tau$ is calculated to satisfy constraints

$$
\mathcal{A} \tau=0,\|\tau\|_{2}=1, \operatorname{det}\left(\begin{array}{c}
\mathcal{A} \\
\tau^{T}
\end{array}\right)>0
$$

and $\alpha=$ const $\in \mathbb{R}_{+}, z(0)=0, \Lambda(0)=0$ the following statements hold:

1. There exists a smooth control trajectory $(u(t), \lambda(t))$ generated by $(15)$ which leaves the point $(u(0), 0)$.

2. The system (13) is transformed by the control law (15) to linear controllable form

$$
H^{(r)}=v
$$

3. The trajectory of $(u(t), \lambda(t))$ either passes through the point $\lambda=1$ or diffeomorphic to a circle.

Proof. 1. The initial statement of the theorem guarantees that the starting point $(u(0), 0)$ of the control trajectory $(u(t), \lambda(t))$ is not attractable. It is easy to see from $(9)$ that $\mathcal{B}=0, \mathcal{A}_{1}=I_{m}(m \times m$ identity matrix $)$ and $\mathcal{A}_{2}=y(0)$ at $t=0$. From $\mathcal{B}=0$ and (15) follows that the control vector $\left(u(0), \lambda^{\left(r_{\max }\right)}(0)\right)=\tau$ is fully determined by the conditions (16). By direct calculations it is easy to see that there are only two values for the $\tau$, satisfying the first two equations in (16), namely

$$
\tau= \pm \frac{1}{N}\left(\begin{array}{c}
-y(0) \\
1
\end{array}\right)
$$

where $N=\left\|(y(0), 1)^{T}\right\|_{2}$. In addition, it is possible to rewrite the last inequality in (16) as follows:

$$
\begin{aligned}
\operatorname{det}\left(\begin{array}{c}
\mathcal{A} \\
\tau^{T}
\end{array}\right) & =\operatorname{det}\left(\begin{array}{cc}
\mathcal{A}_{1} & \mathcal{A}_{2} \\
u(0) & \lambda^{\left(r_{\max }\right)}(0)
\end{array}\right) \\
& =\lambda^{\left(r_{\max }\right)}(0)\left|\mathcal{A}_{1}\right|-u(0)^{T} \mathcal{A}_{2} \\
& =\operatorname{det}\left(\begin{array}{cc}
I_{m} & y(0) \\
\pm \frac{-y(0)}{N} & \pm \frac{1}{N}
\end{array}\right)= \pm \frac{1}{N}\left(1+y(0)^{T} y(0)\right)
\end{aligned}
$$


In order to satisfy this inequality in (16), it is necessary to choose the positive sign in (18) such that $\tau=\frac{1}{N}(-y(0), 1)^{T}$.

Since $\alpha>0$, the condition $\lambda^{\left(r_{\max }\right)}(0)>0$ holds as well and the point $(u(0), 0)$ is not locally stable. The existence and smoothness of the trajectory $(u(t), \lambda(t))$ follows from Lipschitz continuity of the right side (15) and boundedness of $v(t)$.

2. The proof of this statement is straightforward. Note that $\mathcal{A \mathcal { A }}^{+}=I_{m}$. Thus, from (13) one obtains

$$
\begin{aligned}
H^{(r)} & =\mathcal{A}\left(\alpha \tau+\mathcal{A}^{+}(v-\mathcal{B})\right)+\mathcal{B} \\
& =\alpha \mathcal{A} \tau+\mathcal{A A}^{+}(v-\mathcal{B})+\mathcal{B} \\
& =(v-\mathcal{B})+\mathcal{B} \\
& =v .
\end{aligned}
$$

3. This part of the proof will be based on topological arguments used in numerical continuation methods [2]. We will first prove the assertion for the case of relative degree $r_{i}=1$. Note, that in this case we can represent the output $H$ of the augmented system defined in (7) by the following static mapping $H: \mathbb{R}^{m} \times \mathbb{R} \times \mathbb{R}_{+} \rightarrow \mathbb{R}^{m}$

$$
H=H(\xi, \lambda, t), \quad \xi(\bar{t})=\int_{0}^{\bar{t}} u(t) d t .
$$

Differentiating (20) with respect to $t$ results in

$$
\begin{aligned}
\dot{H} & =\frac{\partial H}{\partial t}+\frac{\partial H}{\partial \lambda} \dot{\lambda}+D_{\xi} H \dot{\xi} \\
& =\frac{\partial H}{\partial t}+\frac{\partial H}{\partial \lambda} \dot{\lambda}+D_{\xi} H u,
\end{aligned}
$$

where $D_{\xi} H$ is the Jacobian matrix of $H$ with respect to the state variable $\xi$. On the other hand, from (12) we obtain for $r_{i}=1$

$$
\dot{H}=\mathcal{A}_{1}(x, \lambda) u+\mathcal{A}_{2}(x, z) \dot{\lambda}+\mathcal{B}(x, z, \lambda) .
$$

Comparing (21) and (22) we find that $\mathcal{A}_{1}=D_{\xi} H, \mathcal{A}_{2}=\frac{\partial H}{\partial \lambda}$. The concatenation of $\mathcal{A}_{1}$ and $\mathcal{A}_{2}$ gives $\mathcal{A}=D_{\xi, \lambda} H$. Thus, the condition (14) can be interpreted as follows

$$
\operatorname{rank} D_{\xi, \lambda} H=m
$$


Because of the condition (23) the implicit function theorem can be applied as follows: For any time $t$ there exists a curve $\gamma(t)=(\xi(t), \lambda(t))$ defined by the implicit function $H(\xi, \lambda, t)=0$. The curve $\gamma(t)$ is an one-dimensional manifold and therefore can be either diffeomorphic in coordinates $(\xi, \lambda)$ to a line or to a circle. If $\gamma(t)$ started at $\lambda=0$, then it will intersect $\lambda=1$ if it is diffeomorphic to a line. Alternatively $\gamma(t)$ will turn back before reaching $\lambda=1$ in the case of a circle or turn back when $\lambda>1$ which is also sufficient for the existence of a solution.

Consider now the case $r_{i}>1$. We introduce the integral of $\lambda^{\left(r_{\max }\right)}$ as $\bar{\lambda}(t)=\int_{0}^{t} \lambda^{\left(r_{\max }\right)}(s) d s$ and the static map

$$
\begin{aligned}
\Psi & : \mathbb{R}^{m} \times \mathbb{R} \times \mathbb{R}_{+} \rightarrow \mathbb{R}^{m} \\
H^{(r-1)} & =\Psi(\xi, \bar{\lambda}, t) .
\end{aligned}
$$

By analogy with (20), differentiation of (24) gives

$$
\frac{\partial \Psi}{\partial t}=\mathcal{B}, D_{\xi} \Psi=\mathcal{A}_{1}, \frac{\partial \Psi}{\partial \bar{\lambda}}=\mathcal{A}_{2} .
$$

Applying condition (14) to (24), we obtain that $\operatorname{rank} D_{\xi, \bar{\lambda}} \Psi=m$. Hence, according to the implicit function theorem, arguments similar to the case $r_{i}=1$ can be made for the curve $\gamma(t)=(\xi(t), \bar{\lambda}(t))$, which is defined implicitly by the equation $\Psi(\xi, \bar{\lambda}, t)=0$.

Remark 1. As a result of the feedback control law (15) the augmented system with the output variable (7) is transformed into a linear system (17), which can be controlled by a conventional linear controller. In addition to achieving the desired dynamic behavior this linear control loop can be designed in a robust manner to cope with plant model parametric uncertainty [21].

Remark 2. In general, the dynamics of $\lambda$ generated by (15) is unbounded. The state trajectory $(\lambda(t), x(t))$ starts from the point $\left(0, x_{0}\right)$ and passes the point $\left(1, x^{*}\right)$ where $y=h\left(x^{*}\right)=0$. In order to keep the system in $x^{*}$, in some neighborhood of $(\lambda, x)=\left(1, x^{*}\right)$ the control law (15) is replaced by $\lambda^{\left(r_{\max }\right)}=0$ and $u(x)$ is calculated by an appropriate conventional control law near the point $x^{*}$.

Remark 3. The parameter $\alpha$ in (15) is an additional degree of freedom in controller design. The larger this constant, the faster the solution arrives to $\lambda=1$, but numerical integration becomes more stiff. 
Remark 4. The condition (14) is a standard assumption in the application of parameter continuation methods, which corresponds to the possible existence of limit points of trajectories $(u(t), \lambda(t))$ at which $\mathcal{A}_{1} \notin \operatorname{im} \mathcal{A}_{2}$, and the absence of bifurcation points. At the same time in some regions of phase space $X \times Z$ may be a situation where $\operatorname{rank} \mathcal{A}_{1}(x, \lambda)<m$. In this case, the system with output (7) cannot be directly linearized by the feedback, but the proposed method is still applicable.

Remark 5. Condition (14) can be relaxed a little, but we do not consider this here. In fact, the proposed method allows the existence of the phase space of simple bifurcation points where $\operatorname{dim} \operatorname{ker} \mathcal{A}=2$. When the control trajectory passes through a simple bifurcation point the sign of vector $\tau$ is flipped. A more detailed analysis is given in [2].

Overcoming the bifurcation points where $\mathcal{A}_{1} \in \operatorname{im} \mathcal{A}_{2}$, is also possible within the known approaches for the numerical parameter continuation (e.g., using the Lyapunov-Schmidt decomposition [2]).

Remark 6. Meaning of the parameter $\alpha$ can be demonstrated as follows. Let's consider alone the parameter continuation, putting for external control $v=0$. The function $H$ can be written in the coordinates $(\xi, \bar{\lambda}, t)$ of the control variables integrals, where $\xi(t)=\int_{0}^{t} u(s) d s, \bar{\lambda}=\int_{0}^{t} \lambda^{\left(r_{\max }\right)}(s) d s$.

Overall solution trajectory $(\xi(t), \bar{\lambda}(t), t)$ for the function $H(\xi, \bar{\lambda}, t)=0$ inherits the properties of each particular solution trajectory for stationary continuation problems $H_{t=t_{0}}(\xi, \bar{\lambda})=H\left(\xi, \bar{\lambda}, t_{0}\right)=0$ for every fixed $t_{0}$ if everywhere the direction of change for $\bar{\lambda}$ is collinear with the corresponding component in $\tau$ :

$$
\operatorname{sign}\left(\lambda^{\left(r_{\max }\right)}\right)=\operatorname{sign}\left(\tau_{\lambda}\right),
$$

or $\alpha\left|\tau_{\lambda}\right|>\left|\bar{\tau}_{\lambda}\right|$, which gives the condition for $\alpha$

$$
\alpha>\max _{t}\left|\frac{\bar{\tau}_{\lambda}}{\tau_{\lambda}}\right|
$$

where $\tau_{\lambda}$ is component for $\lambda$ in vector $\tau=\left(\tau_{u} \tau_{\lambda}\right)^{T}$ given by (16) and $\bar{\tau}_{\lambda}$ is variation component for $\lambda$ in $\bar{\tau}=-\mathcal{A}^{+} \mathcal{B}=\left(\bar{\tau}_{u} \bar{\tau}_{\lambda}\right)^{T}$.

Remark 7. The statement 3 of Theorem 1 is given in the traditional manner, by analogy with Theorem (2.1.14) in [2]. It allows that the trajectory could be diffeomorphic to a circle and never reaches $\lambda=1$. We can impose the 
additional condition for initial point:

$$
\operatorname{rank} \mathcal{A}_{1}\left(x_{0}, 0\right)=n .
$$

If this condition satisfied, then the Implicit Function Theorem gives $x$ in terms of $\lambda$ in a neighborhood of $\left(x_{0}, 0\right)$. This implies that solution curve is not diffeomorphic to a circle.

\section{Applications}

\subsection{One Illustrative Example}

In order to illustrate the method we first consider the system (4) and solve the problem 1 starting from $x(0)=1$.

From (4) we obtain

$$
\dot{y}=\left(3 x^{2}-1\right) u .
$$

We now associate with (4) the linear system

$$
\dot{z}=u, \quad \eta=z
$$

with the initial condition $z(0)=0$. From (9) we obtain for the calculation of $\dot{H}$ with (12) the following matrices

$$
\mathcal{A}_{1}=\lambda\left(3 x^{2}-1\right)+1-\lambda, \mathcal{A}_{2}=y-\eta, \mathcal{B}=0 .
$$

Using this result and the feedback equation (see Remark 1)

$$
v=-k H,
$$

the control action can be calculated from (15) as follows

$$
\begin{aligned}
\left(\begin{array}{l}
u \\
\dot{\lambda}
\end{array}\right) & =\alpha \tau+\mathcal{A}^{+}(v-\mathcal{B}) \\
& =\alpha \tau-k\left(\lambda\left(3 x^{2}-1\right)+1-\lambda \quad y-\eta\right)^{+} H .
\end{aligned}
$$

Complete stabilization problem for this particular example could be realized accordingly to Remark 2 as follows. If control trajectory approached a 
neighborhood of $\lambda=1$, then fix $\lambda:=1$ and stabilizing controller could be implemented via feedback linearization with decoupling term obtained earlier in (5):

$$
u=\frac{v}{3 x^{2}-1}=\frac{-k x}{3 x^{2}-1} .
$$

Thus we have following one-step control rule: if $\lambda<1-\epsilon$ then apply control (29), if $\lambda>1-\epsilon$ then fix $\lambda=1$ and apply control (30), where $\epsilon>0$ sufficiently small positive constant.

The simulation results obtained with this Simulink model for $k=10$ and $\alpha=5$ are given in Figure 1 and illustrate the transition of the output $y$ to 0 while $\lambda$ moves from 0 to 1 .

Additional simulations were conducted to demonstrate performance of the method in case of model parametric uncertainty. The control was synthesized using approximate plant model in form

$$
\dot{\bar{x}}=u, \quad \bar{y}=\bar{x}\left(\bar{x}^{2}-\frac{1}{2}\right)+\frac{1}{2}, \quad \bar{x}(0)=1 .
$$

The simulation results indicated that outer loop robustifies control performance and the desired output value $y=0$ was asymptotically reached due to external proportional feedback $v=-k H$ even in case of model parameter uncertainty.

Finally, the simulations illustrate the change in the dynamic behavior at the points $x=\frac{1}{\sqrt{3}}$ and $x=-\frac{1}{\sqrt{3}}$ marked by the red dotted lines where conventional feedback linearization is not feasible.

\subsection{Three Phase Induction Motor Control}

Consider the application of the proposed method for controlling the speed and flux linkage of an induction machine. As described in [6] the three-phase induction machine is an example of a system that cannot be linearized by state feedback as the decoupling matrix of the feedback transformation is singular if the rotor flux linkage is zero which occurs at the startup of the motor. In this 

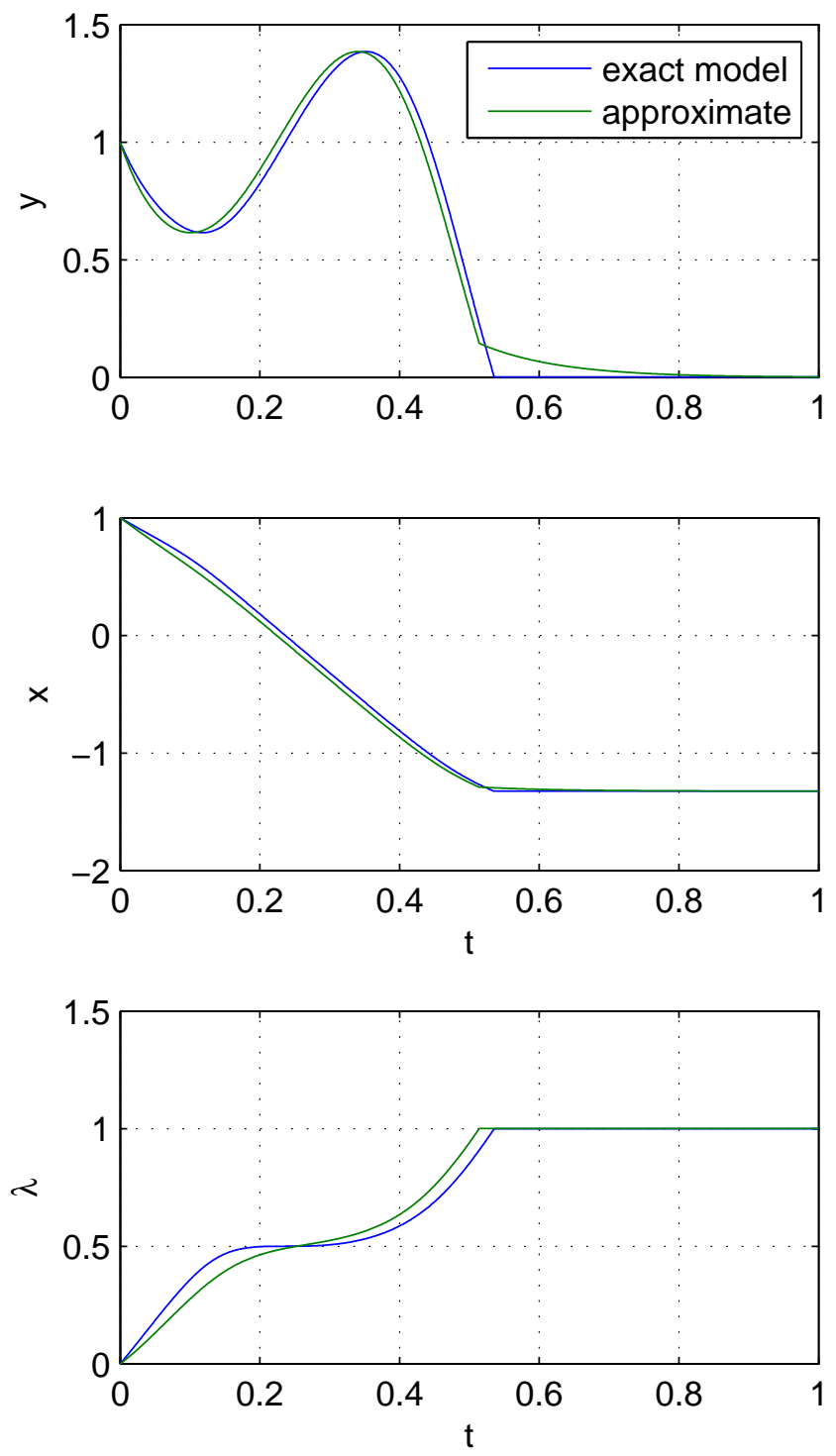

Figure 1: Simulation results for the simple system control. 
design procedure we use the system model given in [11]:

$$
\begin{aligned}
& \dot{\omega}=\mu \phi_{r} i_{s q}-\frac{p T_{m}}{J} \\
& \dot{\phi}_{r}=-\tau_{r}^{-1} \phi_{r d}+\tau_{r}^{-1} M_{s r} i_{s d} \\
& \dot{i}_{s d}=\beta \tau_{r}^{-1} \phi_{r}-\tau_{1}^{-1} i_{s d}+\omega_{s} i_{s q}+\frac{u_{s d}}{L_{1}} \\
& \dot{i}_{s q}=-\beta \omega \phi_{r}-\tau_{1}^{-1} i_{s q}-\omega_{s} i_{s d}+\frac{u_{s q}}{L_{1}} .
\end{aligned}
$$

The state variables are the rotor angular speed $\omega$, the rotor flux linkage $\phi_{r}$ and the stator current projections on the $d q$-axis reference frame $i_{s d}, i_{s q}$. Control variables are the stator voltages $u_{s d}$ and $u_{s q} . T_{m}$ is the load torque and is considered as disturbance. The model parameters are given based on the stator and rotor resistances and self-inductances $R_{s}, L_{s}$ and $R_{r}, L_{r}$, the mutual inductance $M_{s r}$ and the number of pole pairs $p$ :

$$
\begin{aligned}
& \tau_{r}=\frac{L_{r}}{R_{r}}, \quad \quad \mu=p^{2} \frac{M_{s r}}{J L_{r}}, \quad \beta=\frac{M_{s r}}{L_{r} L_{1}}, \\
& L_{1}=L_{s}-\frac{M_{s r}^{2}}{L_{r}}, \quad R_{1}=R_{s}+R_{r}\left(\frac{M_{s r}}{L_{r}}\right)^{2}, \quad \tau_{1}=\frac{L_{1}}{R_{1}} .
\end{aligned}
$$

Due to rotor flux orientation the synchronous rotor angular speed $\omega_{s}$ is given by

$$
\omega_{s}=\omega+\frac{M_{s r} i_{s q}}{\tau_{r} \phi_{r}} .
$$

The control objective is to control the mechanical rotor speed $\omega / p$ and the rotor flux linkage $\phi_{r}$.

We will use the approach from [19] for controlling the system given by (32) based on two feedback loops. The internal loop controls the currents $i_{s d}, i_{s q}$ using two PI-controllers and decoupling of the two current component dynamics by

$$
\begin{aligned}
& u_{s d}=L_{1}\left(\nu_{s d}-\omega_{s} i_{s q}\right) \\
& u_{s q}=L_{1}\left(\nu_{s q}+\beta \omega \phi_{r}+\omega_{s} i_{s d}\right) .
\end{aligned}
$$

As a result we obtain a linear dynamics for the currents given by

$$
\begin{aligned}
& \dot{i}_{s d}=\nu_{s d}+\beta \tau_{r}^{-1} \phi_{r}-\tau_{1}^{-1} i_{s d} \\
& \dot{i}_{s q}=\nu_{s q}-\tau_{1}^{-1} i_{s q} .
\end{aligned}
$$


The controllers for the current dynamics given by (35) and (36) are designed such that the controlled current dynamics can be neglected in the design process of the outer loop [19]. The external loop controls the mechanical rotor speed and the rotor flux linkage. We use PI-controllers such that the structure of the system resembles a classical FOC-control with the only difference that the output of each PI-controller in the outer loop is passed through an appropriate nonlinear coordinate transformation. For applying the new method we consider as output variables the deviation from the given reference value of the mechanical speed and the rotor flux linkage, respectively

$$
y_{1}=\frac{\omega-\omega_{\mathrm{Ref}}}{p} \quad y_{2}=\phi_{r}-\phi_{r, \operatorname{Ref}} .
$$

The first differentiation of the outputs $y_{1}$ and $y_{2}$ yields

$$
\begin{aligned}
& \dot{y}_{1}=\frac{\mu \phi_{r} i_{s q}}{p} \\
& \dot{y}_{2}=-\tau_{r}^{-1} \phi_{r}+\tau_{r}^{-1} M_{s r} i_{s d} .
\end{aligned}
$$

Let us associate with (37) and (38) a linear system of a form

$$
\dot{\eta}_{1}=u_{1}=i_{s q}, \quad \dot{\eta}_{2}=u_{2}=i_{s d}, \quad \eta(0)=(0,0)^{T} .
$$

Using (9) and (11), we can write the following equation for the augmented system:

$$
\begin{aligned}
H & =(1-\lambda) \eta+\lambda y \\
\dot{H} & =\lambda \dot{y}+u(1-\lambda)+\dot{\lambda}(y-\eta) \\
\dot{H}_{1} & =\lambda \mu \phi_{r} i_{s q} / p+i_{s q}(1-\lambda)+\dot{\lambda}\left(y_{2}-\eta_{2}\right) \\
\dot{H}_{2} & =\lambda\left(-\tau_{r}^{-1} \phi_{r}+\tau_{r}^{-1} M_{s r} i_{s d}\right)+i_{s d}(1-\lambda)+\dot{\lambda}\left(y_{1}-\eta_{1}\right) .
\end{aligned}
$$

According to Theorem 1, the augmented plant (39)-(40) can be transformed into $\dot{H}=v$ by the feedback (15)

$$
\begin{aligned}
\left(\begin{array}{c}
u \\
\dot{\lambda}
\end{array}\right) & =\alpha \tau+\mathcal{A}^{+}(v-\mathcal{B}) \\
\mathcal{A} & =\lambda\left(\begin{array}{ccc}
\frac{\mu \phi_{r}}{p} & 0 & y_{1}-\eta_{1} \\
0 & \frac{M_{s r}}{\tau_{r}} & y_{2}-\eta_{2}
\end{array}\right)+(1-\lambda)\left(\begin{array}{lll}
1 & 0 & 0 \\
0 & 1 & 0
\end{array}\right) \\
\mathcal{B} & =\lambda\left(\begin{array}{c}
0 \\
-\frac{\phi_{r}}{\tau_{r}},
\end{array}\right)
\end{aligned}
$$


where $i_{s q}=u_{1}, i_{s d}=u_{2}$.

After transforming the system (39)-(40) to $\dot{H}=v$ it is necessary to implement an external linear control loop, which keeps $y$ at the desired setpoints for $\lambda=1$ when $H=y$. The variables $v_{1}$ and $v_{2}$ correspond to the output of the two PI-controllers in the outer loop.

Note that for $\lambda=1$ the linearizing transformation (41) reduces into following form

$$
\begin{aligned}
i_{s d} & =\frac{\tau_{r}}{M_{s r}}\left(v_{1}+\tau_{r}^{-1} \phi_{r}\right) \\
i_{s q} & =\frac{p}{\mu \phi_{r}} v_{2}
\end{aligned}
$$

which is conventional feedback linearization applied for motor control after fixing $\lambda:=1$ according to Remark 2 .

During the simulation Gaussian noise with variance 0.005 obtained from a source of pseudorandom numbers was added to the stator current components $i_{s d}$ and $i_{s q}$ which corresponds to $15 \mathrm{~mA}$ random error of current measurement. The parameter $\alpha$ was set to $\alpha=20$ and stator voltages $u_{s d}, u_{s q}$ are limited to the interval [-200, $200 \mathrm{~V}]$.

$\begin{array}{lrll}P & 4 & \mathrm{~kW} & \text { rated power } \\ M_{s r} & 0.175 & \mathrm{H} & \text { mutual inductance } \\ R_{s} & 1.2 & \Omega & \text { stator resistance } \\ R_{r} & 0.873 & \Omega & \text { rotor resistance } \\ L_{s} & 0.195 & \mathrm{H} & \text { stator inductance } \\ L_{r} & 0.195 & \mathrm{H} & \text { rotor inductance } \\ J & 0.013 & \mathrm{kgm}^{2} & \text { motor and load inertia } \\ p & 2 & \text { pole pairs } & \end{array}$

Table 1: Motor parameters.

The simulation results shown in Figures 2 illustrate the performance of the control algorithm during the first second after startup. The control objective is to follow the reference acceleration $\operatorname{ramp} \omega_{\text {Ref }}(t)=65 t \mathrm{rad} / \mathrm{s}$ and maintain $\phi_{r}$ at the level of $\phi_{r, \text { Ref }}(t)=0.31 \mathrm{Vs}$. The motor operates under a load torque $T_{m}=2 \mathrm{Nm}$. Initial undershoot in speed $\omega$ is caused by external disturbance application of load torque.

From the simulation results we observe the smooth transition from $\lambda=0$ to $\lambda=1$ in the interval $t \in\left[\begin{array}{ll}0 & 0.15 \mathrm{~s}\end{array}\right]$. While the rotor flux linkage $\phi_{r}$ reaches the reference value, the mechanical rotor speed first moves in the opposite direction due to the applied load torque $T_{m}$ and then reaches the desired trajectory. 

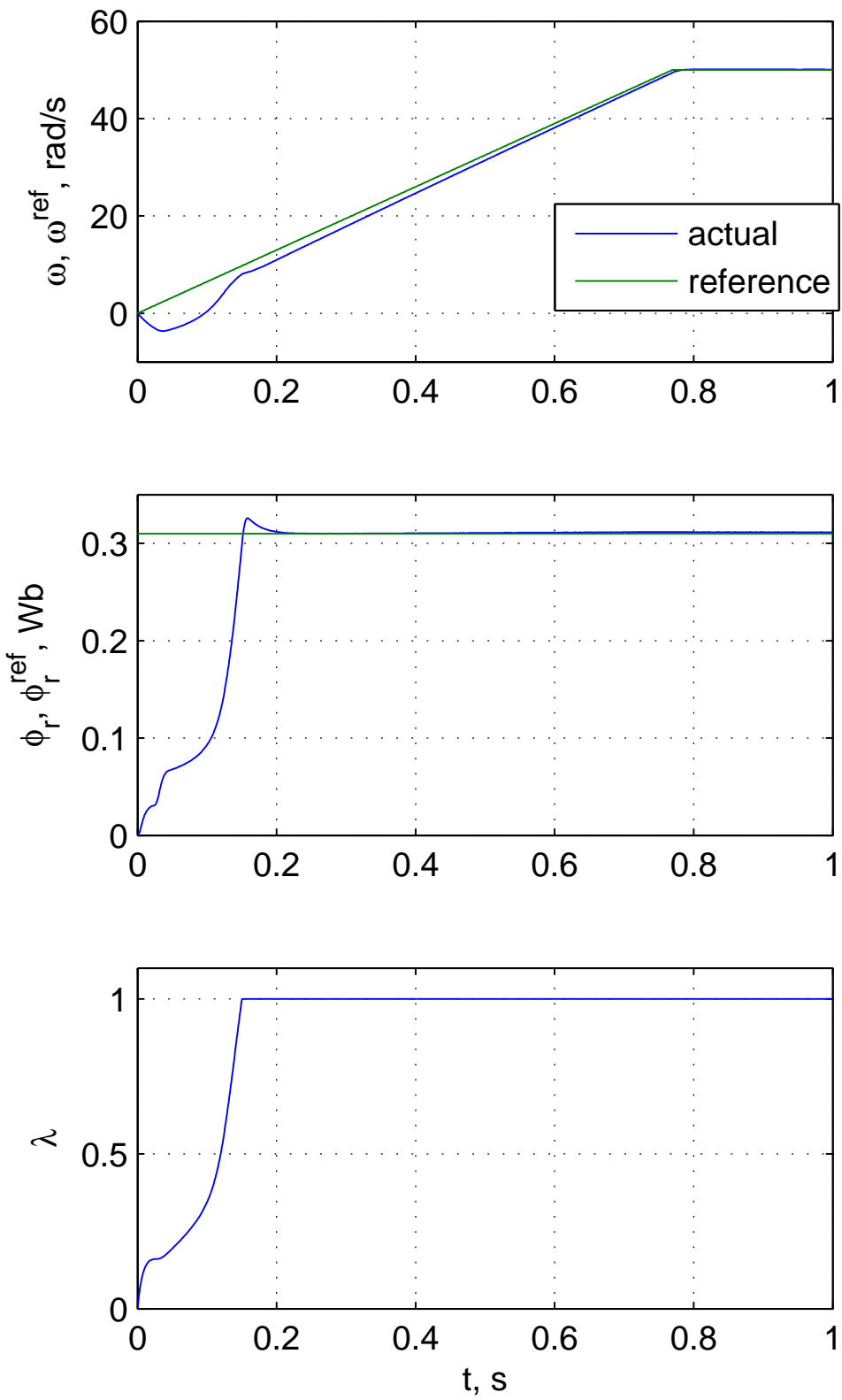

Figure 2: Simulation results for the induction motor control. 


\section{Conclusion}

In this paper we propose a new method for controlling affine nonlinear systems. Starting from the conceptual simplicity of feedback linearization this new method expands the scope of their applicability to irregular systems with poorly expressed relative degree. The main idea is to augment the system in such a way that the augmented system can be controlled by feedback linearization. Currently, the dynamics of the parameter $\lambda$ is unbounded, such that in the neighborhood of the desired output value a switch of the control law is necessary.

The method is illustrated on a simple SISO-system and by controlling the speed and the rotor flux linkage in a three-phase induction machine.

Future work will focus on methods to eliminate the need for this switching. In addition, the modeling of the influence of uncertainties in an explicit form will be investigated and transient performance of the augmented system will be analyzed formally as well. Also generalization for case $r<n$ and impact of zero dynamics will be investigated.

\section{References}

[1] C. Aguilar-Ibañez, M. S. Suarez-Castanon, and J. d. J. Rubio, Stabilization of the ball on the beam system by means of the inverse Lyapunov approach, Mathematical Problems in Engineering (2012), ID 810597.

[2] E. L. Allgower and K. Georg, Introduction to Numerical Continuation Methods, SIAM (2003).

[3] E. Asarin, T. Dang, and A. Girard, Hybridization methods for the analysis of nonlinear systems, Acta Informatica, 43, No. 7 (2007), 451-476.

[4] D. A. Barton and S. G. Burrow, Numerical continuation in a physical experiment: investigation of a nonlinear energy harvester, Journal of Computational and Nonlinear Dynamics, 6, No. 1 (2011), 011010.

[5] D. A. Barton, B. P. Mann, and S. G. Burrow, Control-based continuation for investigating nonlinear experiments, Journal of Vibration and Control, 18, No. 4 (2012), 509-520.

[6] M. Bodson and J. Chiasson, Differential-geometric methods for control of electric motors, International Journal of Robust and Nonlinear Control, 8, No. 11 (1998), 923-954. 
[7] A. Borisevich and M. Krupskaya, Some aspects of numerical continuation methods in control of nonlinear affine systems, In Proceedings of the International Symposium of Applied Natural Sciences, 111-115. UCM Trnava (2011).

[8] A. Borisevich and G. Schullerus, Switching strategy based on homotopy continuation for non-regular affine systems with application in induction motor control, arXiv Preprint 1203.5919 (2012).

[9] W.-H. Chen and D. J. Ballance, On a switching control scheme for nonlinear systems with ill-defined relative degree, Systems and Control Letters, 47, No. 2 (2002), 159-166.

[10] A. Choukchou-Braham, B. Cherki, and M. Djemaı, Stabilisation of a class of underactuated system with tree structure using backstepping approach, Nonlinear Dynamics and Systems Theory (2012), 345.

[11] A. Fekih and F. N. Chowdhury, On nonlinear control of induction motors: comparison of two approaches, In: American Control Conference, 2004. Proceedings of the 2004, Volume 2, IEEE (2004), 1135-1140.

[12] V. Hagenmeyer and E. Delaleau, Exact feedforward linearization based on differential flatness, International Journal of Control, 76, No. 6 (2003), 537-556.

[13] A. Isidori, Nonlinear Control Systems, Volume 1, Springer (1995).

[14] M.-F. Jang and Y.-S. Chou, Robust control strategies for a class of nonlinear systems with an ill-defined relative degree, Journal of the Taiwan Institute of Chemical Engineers, 44, No. 5 (2013), 734-742.

[15] Y. Lenoir, P. Martin, and P. Rouchon, $2 \mathrm{k} \pi$, the juggling robot, In Decision and Control, 1998. Proceedings of the 37th IEEE Conference on, Volume 2 (1998), 1995-2000.

[16] A. Lotfazar and M. Eghtesad, Application and comparison of passivitybased and integrator backstepping control methods for trajectory tracking of rigid-link robot manipulators incorporating motor dynamics, International Journal of Robotics and Automation, 22, No. 3 (2007), 196-205.

[17] R. Mahony, I. Mareels, G. Bastin, and G. Campion, Output stabilization of square nonlinear systems, Automatica, 33, No. 8 (1997), 1571-1577. 
[18] P. Martin, P. Rouchon, and R. Murray, Flat systems, equivalence and trajectory generation, Technical Report, California Institute of Technology (2003).

[19] K. Mohanty and N. De, Nonlinear controller for induction motor drive, In: Industrial Technology 2000. Proceedings of IEEE International Conference on, Volume 1 (2000), 382-387.

[20] H. Nijmeijer and A. Van der Schaft, Nonlinear Dynamical Control Systems, Springer (1990).

[21] S. Palanki, J. C. Cockburn, and S. N. Kolavennu, Robust state feedback synthesis for control of non-square multivariable nonlinear systems, Journal of Process Control, 13, No. 7 (2003), 623-631.

[22] A. Pisano and E. Usai, Sliding mode control: A survey with applications in math, Mathematics and Computers in Simulation, 81, No. 5 (2011), 954-979.

[23] S. Prajna, A. Papachristodoulou, and F. Wu, Nonlinear control synthesis by sum of squares optimization: A Lyapunov-based approach, In The 5th Asian Control Conference (ASCC 2004), volume 1, (2004) 157-165.

[24] I. Siller-Alcalá, J. Liceaga-Castro, R. Alcántara-Ramırez, and J. JaimesPonce, Speed nonlinear predictive control of a series DC motor for bidirectional operation, Proceedings of the 13th IASME/WSEAS (2011), 182-187. 
